THE EUROPEAN PHYSICAL JOURNAL PLUS

(ITALIAN PHYSICAL SOCIETY)

ISSN: 2190-5444

PUBLISHER: SPRINGER

IMPACT FACTOR= 1.521

Accepted May $16^{\text {th }} 2017$

\title{
Variable-Viscosity Thermal Hemodynamic Slip Flow Conveying Nanoparticles through a Permeable-Walled Composite Stenosed Artery ${ }^{1}$ Noreen Sher Akbar, ${ }^{2 \#}$ Dharmendra Tripathi and ${ }^{3} \mathrm{O}$. Anwar Bég \\ ${ }^{1}$ DBS\&H CEME National University of Sciences and Technology, Islamabad, Pakistan \\ ${ }^{2}$ Department of Mechanical Engineering, Manipal University Jaipur, Rajasthan-303007, India. \\ ${ }^{3}$ Fluid Mechanics, Aeronautical and Mechanical Engineering, University of Salford, Newton \\ Building, The Crescent, Salford, Manchester, M5 4WT, UK.
}

\begin{abstract}
This paper presents a mathematical model for simulating viscous, incompressible, steady-state blood flow containing copper nanoparticles and coupled heat transfer through a composite stenosed artery with permeable walls. Wall slip hydrodynamic and also thermal buoyancy effects are included. The artery is simulated as an isotropic elastic tube, following Joshi et al (2009), and a variable viscosity formulation is employed for the flowing blood. The equations governing the transport phenomena are non-dimensionalized and the resulting boundary value problem is solved analytically in the steady state subject to physically appropriate boundary conditions. Numerical computations are conducted to quantify the effects of relevant hemodynamic, thermophysical and nanoscale parameters emerging in the model on velocity and temperature profiles, wall shear stress, impedance resistance and also streamline distributions. The model may be applicable to drug fate transport modeling with nanoparticle agents and also the optimized design of nanoscale medical devices for diagnosing stenotic diseases in circulatory systems.
\end{abstract}

Keywords: Blood flow; Copper Nanofluid; Composite Stenosed Arteries, Permeable Wall, Drug Delivery.

${ }^{\#}$ Corresponding author-email: dharmendra.tripathi@jaipur.manipal.edu 


\section{INTRODUCTION}

The significant complexity of blood flow and the ever-increasing incidence of cardiovascular diseases, continue to stimulate significant interest in developing improved medication delivery and infusion devices. Often blood vessels (arteries and capillaries) or other tubular physiological organs can become abnormally narrow, a characteristic known in physiology and medical engineering literature as stenosis. Stenoses are usually caused by atherosclerosis, a condition where a blood vessel supplying blood to the brain (and other organs) is narrowed due to fatty deposits, known as plaques, on the interior walls of the vessel. Several factors influence how stenosis can be treated, including the percentage of blood vessel blockage and the patient's overall risk of a first or second stroke. Although numerous clinical studies of stenotic blood flows have been communicated, considerable work has also emerged in fluid dynamic simulation (theoretical and numerical) in this intriguing area of medical hydrodynamics. An excellent overview of this field has been given by Berger and Jou [1]. Misra et al. [2] presented an analytical study of blood flow through an arterial segment having a mild stenosis, simulating the artery as a thin-walled and initially stressed orthotropic non-linear viscoelastic cylindrical tube filled with a non-Newtonian fluid representing blood. Ellahi et al.[3] investigated the unsteady flow of non-Newtonian blood through composite stenosis with Eringen's micropolar fluid. Akbar et al. [4] derived closed-form solutions for nanofluid flow through composite stenosed arteries with permeable walls. Akbar et al. [5] further discussed the nanostructure effects on the physiological flow of CNT nanofluids where base fluid is considered as viscous fluid of temperature dependent variable viscosity. They observed that the velocity of CNT fluid diminishes with increasing the Grashof number. Mekheimer et al. [6] mathematically studied the blood flow through an elastic artery with overlapping stenosis under the effect of induced magnetic field. Ellahi et al. [7] investigated theoretically the transient blood flow of a Jeffery viscoelastic fluid through two types of arteries, namely (i) composite stenosed artery and (ii) anisotropically tapered stenosed artery with permeable walls. Chakravarty and Sannigrahi [8] discussed the flow-field in a porous stenotic artery when it is subjected to a single cycle of body acceleration and the simulated artery is taken as an isotropic elastic tube containing a viscous incompressible fluid representing the flowing blood. Nadeem and Ijaz [9] studied the physical characteristics of blood flow in the presence of stenosis and nanoparticles through a curved 
channel with permeable walls. Back et al. [10] measured the pressure distributions through a hollow vascular axisymmetric replica of a segment of the left circumflex human coronary artery with the mildly atherosclerotic diffuse disease. Chakraborty et al. [11] studied blood flow through an inclined mild stenosis artery with slip present at the stenotic wall and blood analyzed as a particle-fluid suspension. Owida et al. [12] suggested vascular grafts that are small in diameter as an initiating factor for the progress of wall thickening along the suture line, observing that they tend to occlude rapidly. Schneiderman et al. [13] simulated the oxygen transfer in fully developed, pulsating, laminar flow in rigid and distensible tubes. Mekheimer et al. [14] deployed a particle-fluid suspension model for the axisymmetric blood flow through curved coaxial tubes where the outer tube has a mild overlapping stenosis while the inner tube is uniform and rigid and represents a catheter. Akbar et al. [15] investigated theoretically the unsteady rheological blood flow fluid through composite stenosed arteries with permeable walls, using the Williamson viscoelastic model. Mollica et al. [16] demonstrated that tumor blood flow (TBF) plays a fundamental role in tumor growth and treatment, and is characterized by spatial and temporal heterogeneities. Joshi et al. [17] discussed the porosity effects on Boundary Layer Bodewadt Flow of magnetic nanofluids with geothermal viscosity. They observed that with increasing the magnitude of Prandtl number from 20 to 80, the heat transfer rate increases by 93.35\%. Manimaran [18] presented detailed numerical simulations for rheological blood flow with a power-law model, considering arterial stenoses with $48 \%$ areal occlusion., observing that pressure drop across a stenosed artery is insignificantly modified by surface irregularities at low Reynolds numbers, whereas a substantial influence is computed at higher Reynolds numbers. Rabby et al. [19] investigated computationally the non-Newtonian hemodynamics in unsteady periodic flows in a two-dimensional vessel with two idealized stenoses of $75 \%$ and $50 \%$ degrees, respectively, employing four different rheological models (Carreau, Cross, modified Casson and Quemada constitutive equations). They presented extensive visualizations for streamwise velocity, pressure distribution, and wall shear stress (WSS) as well as the vorticity and examined carefully the recirculation zones at the post-stenotic region. They noted that the risk of thrombogenesis is minimized at the downstream location from the stenoses but that reduced blood supply is predicted in the Newtonian model relative to the non-Newtonian models. Akber et al. [20] reported the nanofluids flow driven by the combined effects of 
magnetohydrodynamics and peristalsis in presence of velocity and slip effects. Two types of nanoparticles ( $\mathrm{Ag}$ and $\mathrm{SiO} 2)$ have been considered. They found that the pressure gradient for $\mathrm{Ag}$ nanofluid is minimum and it is maximum for pure water. That means the nanoparticles oppose the peristaltic flow however they enhance the thermal conductivity of base fluid. Molla and Paul [21] investigated computationally the 3-D pulsatile transition-to-turbulent non-Newtonian blood flow through an arterial stenosis with five different blood viscosity models including the modified-Casson model and a large eddy simulation numerical code. They evaluated the influence of different rheology on global maximum shear rate, post-stenotic re-circulation zone, mean shear stress, mean pressure, and turbulent kinetic energy. They observed that the nonNewtonian formulations increased significantly the length of the post-stenotic re-circulation region by a displacement of the shear layer reattachment point, whereas the turbulent kinetic energy at the immediate post-lip of the stenosis was reduced with greater non-Newtonian viscosity. More recently Zaman et al. [22] studied combined heat and mass diffusion in nonNewtonian blood flow in an overlapping stenosed artery. They used the Cross viscoelastic model and observed that the velocity, temperature, and species concentration fields are substantially modified with Weissenberg viscoelastic number, Brinkman and also Soret (thermo-diffusive) number, over a range of Prandtl numbers. Neofytou and Drikakis [23] studied the influence of non-Newtonian behaviour on unsteady periodic flows in a two-dimensional channel with a stenosis, deploying Casson, power-law and Quemada models, and observing that several vortices downstream of the stenosis arise and that the strength and location of vortex formation, wall shear stress magnitudes and separation behind the stenosis is greatly affected by the specific rheology considered. Further studies of peristaltic flow in presence electroosmosis phenomenon using rheological models (couple stress fluids) have been presented by, for example, Tripathi et al. [24] who observed that the physiological flow may modulate with applying the external electric field.

The above studies have generally not considered nanoscale effects, despite the important potential of nanoparticles in pharmacodynamics, treatment of ailments and other applications in medicine [25-29]. Motivated by such developments in the current investigation we present a new model for blood flow employing a variable-viscosity copper-nanofluid formulation. Heat transfer effects are also considered including thermal buoyancy and heat generation. The geometry 
studied comprises a composite stenosed artery with permeable walls. Hydrodynamic wall slip conditions are also taken into account as these have been identified as being significant in certain clinical situations[30]. Analytical solutions are developed for the transformed boundary value problem. The influence of key thermophysical, nanoscale and hemodynamic parameters on velocity, temperature, wall shear stress, streamline distributions and impedance are examined in detail. Considering the wide applications of nanofluids flow, most recently, some relevant works [31-35] on nanofluid flow with various applications have been reported in the literature.

\section{MATHEMATICAL MODEL FOR NANOFLUID HEMODYNAMICS}

Consider an axisymmetric flow of blood through a vertical composite stenosis in a circular tube of finite length, $L$, with permeable wall as shown in Fig.1. Gravity is therefore invoked in the physics. The geometry of arterial wall with composite stenosis is described by Joshi et al. [36] as:

$$
\frac{R(z)}{R_{0}}=\left\{\begin{array}{lr}
1-\frac{2 \delta}{R_{0} L_{0}}(z-d) ; & d<z \leq d+\frac{L_{0}}{2}, \\
1-\frac{\delta}{2 R_{0}}\left(1+\cos \frac{2 \pi}{L_{0}}\left(z-d-\frac{L_{0}}{2}\right)\right) ; & d+\frac{L_{0}}{2}<z \leq d+L_{0} \\
1 ; & \text { otherwise. }
\end{array}\right.
$$

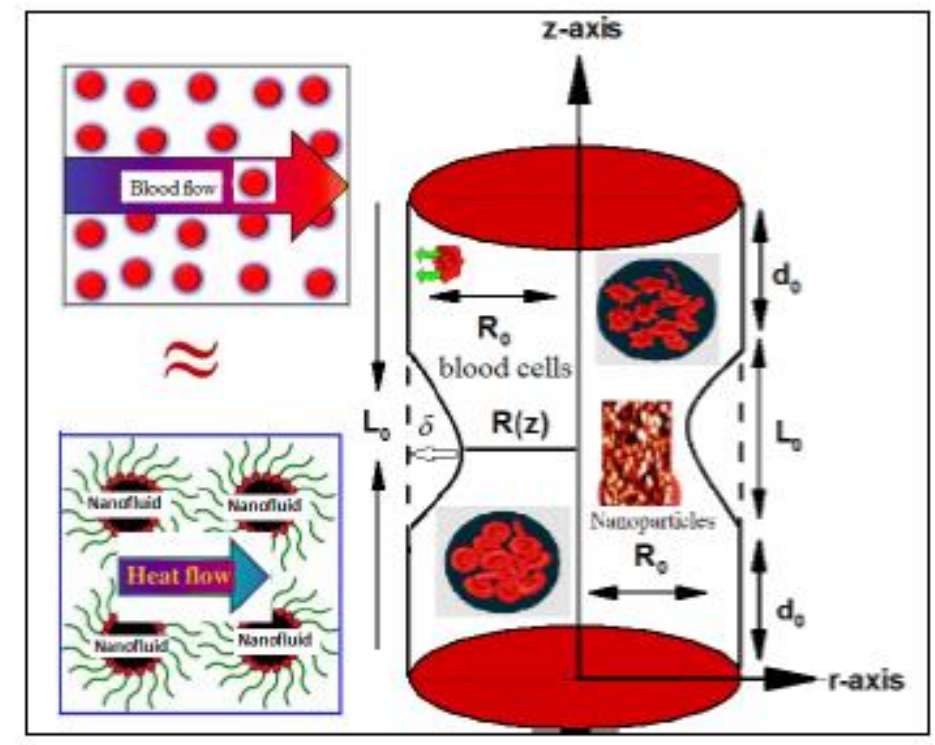

Fig.1. Geometry of the nanofluid blood flow through a composite stenosed artery. 
where $R(z)$ is the radius of the artery in the obstructed region, $R_{0}$ designates the radius of normal artery, $L_{0}, d, \delta$ denote the length, location and height of the stenosis, respectively. The governing equations for mass, momentum and heat conservation for an incompressible variableviscosity nanofluid with heat source present, can be written as:

$\frac{1}{r} \frac{\partial(r v)}{\partial r}+\frac{\partial u}{\partial z}=0$

$\rho_{n f}\left(v \frac{\partial v}{\partial r}+u \frac{\partial v}{\partial z}\right)=-\frac{\partial p}{\partial r}+\frac{\partial}{\partial r}\left(2 \mu_{n f}(T) \frac{\partial v}{\partial r}\right)+\frac{\partial}{\partial z}\left(\mu_{n f}(T)\left(2 \frac{\partial v}{\partial z}+\frac{\partial u}{\partial r}\right)\right)$

$\rho_{n f}\left(v \frac{\partial u}{\partial r}+u \frac{\partial u}{\partial z}\right)=-\frac{\partial p}{\partial z}+\frac{\partial}{\partial z}\left(2 \mu_{n f}(T) \frac{\partial u}{\partial z}\right)+\frac{1}{r} \frac{\partial}{\partial r}\left[\mu_{n f}(T) r\left(\frac{\partial v}{\partial z}+\frac{\partial u}{\partial r}\right)\right]$

$$
+\rho_{n f} g \alpha\left(\bar{T}-T_{0}\right)
$$

$\left(\rho_{c p}\right)_{n f}\left(v \frac{\partial T}{\partial r}+u \frac{\partial T}{\partial z}\right)=k_{n f}\left[\frac{\partial^{2} T}{\partial r^{2}}+\frac{1}{r} \frac{\partial T}{\partial r}+\frac{\partial^{2} T}{\partial z^{2}}\right]+Q_{0}$,

The appropriate boundary conditions prescribed are:

$\frac{\partial u}{\partial r}=0, \quad \frac{\partial T}{\partial r}=0 \quad$ at $\quad r=0$

$u=u_{B}, \quad T=0$, at $r=\frac{R(z)}{R_{0}}$.

Here $r$ and $z$ are the coordinates, $z$ is taken as the center line (longitudinal axis) of the tube and $r$ transverse to it, $u_{B}$ represents the wall slip velocity, $u$ and $v$ are the velocity components in $r$ and $z$ directions, respectively, $T$ is the local temperature of the fluid. Also, $\rho_{n f}$ is the effective density, $\mu_{n f}$ is the effective dynamic viscosity, $\left(\rho c_{p}\right)_{n f}$ is the heat capacitance, $\alpha_{n f}$ is the effective thermal diffusivity and $k_{n f}$ is the effective thermal conductivity of the nanofluid, which are defined as follows: 


$$
\begin{aligned}
\rho_{n f} & =(1-\varphi) \rho_{f}+\varphi \rho_{f}, \mu_{n f}=\frac{\mu_{f}(\theta)}{(1-\varphi)^{2.5}} \\
\left(\rho c_{p}\right)_{n f} & =(1-\varphi)\left(\rho c_{p}\right)_{f}+\varphi\left(\rho c_{p}\right)_{s}, \alpha_{n f}=\frac{k_{n f}}{\left(\rho c_{p}\right)_{n f}}, \\
k_{n f} & =k_{f}\left(\frac{k_{s}+2 k_{f}-2 \varphi\left(k_{f}-k_{s}\right)}{k_{s}+2 k_{f}+2 \varphi\left(k_{f}-k_{s}\right)}\right) .
\end{aligned}
$$

Proceeding with the analysis, to facilitate analytical solutions, we introduce the following nondimensional variables:

$$
\begin{aligned}
\bar{r} & =\frac{r}{R_{0}}, \bar{z}=\frac{z}{L_{0}}, \bar{v}=\frac{L_{0}}{\delta U} v, \bar{u}=\frac{u}{U}, \bar{d}=\frac{d}{L_{0}}, \bar{R}=\frac{R}{R_{0}}, \\
G_{r} & =\frac{g \alpha R_{0}^{2} T_{0} \rho_{n f}}{U \mu_{f}}, \bar{\delta}=\frac{\delta}{R_{0}}, \theta=\frac{T-T_{0}}{T_{0}}, \bar{p}=\frac{U L_{0} \mu}{R_{0}^{2}} p, \\
\beta & =\frac{Q_{0} R_{0}^{2}}{k_{f} T_{0}}
\end{aligned}
$$

where $U$ is the velocity averaged over the section of the tube with radius $R_{0}$. Invoking the variables defined in eq.(8) in eqs. $(2-5)$ and applying the additional condition $\varepsilon=\frac{R_{0}}{L_{0}}=\mathrm{O}(1)$ for the case of a mild stenosis $\left(\frac{\delta}{R_{0}}<<1\right)$, the non-dimensional governing equations after dropping the dashes assume the following form:

$\frac{\partial p}{\partial r}=0$

$\frac{\partial p}{\partial z}=\frac{1}{r} \frac{\partial}{\partial r}\left(\frac{\mu_{n f}(\theta)}{\mu_{0}} r \frac{\partial u}{\partial r}\right)+G_{r}\left(\frac{(\rho \gamma)_{n f}}{(\rho \gamma)_{f}}\right) \theta$,

$0=\frac{1}{r} \frac{\partial}{\partial r}\left(r \frac{\partial \theta}{\partial r}\right)+\beta\left(\frac{k_{s}+2 k_{f}+2 \varphi\left(k_{f}-k_{s}\right)}{k_{s}+2 k_{f}-2 \varphi\left(k_{f}-k_{s}\right)}\right)=0$,

$\frac{\mu_{n f}(\theta)}{\mu_{0}}=\frac{e^{-\Lambda \theta}}{(1-\phi)^{2.5}}, \quad e^{-\Lambda \theta}=1-\alpha \Lambda \theta+O\left(\Lambda^{2}\right)$,

where $\Lambda, \beta$ and $G_{r}$ are the viscosity parameter, heat absorption (source) parameter and Grashof (thermal buoyancy effect) number, respectively. The non-dimensional boundary conditions for 
velocity and temperature at the permeable wall become:

$$
\begin{aligned}
& \frac{\partial u}{\partial r}=0, \frac{\partial \theta}{\partial r}=0 \quad \text { at } \quad r=0, \\
& u=u_{B}, \frac{\partial u}{\partial r}=\frac{\alpha}{\sqrt{D_{a}}}\left(u_{B}-u_{\text {porous }}\right), \theta=0 \quad \text { at } r=R(z),
\end{aligned}
$$

where $u_{B}$ is the slip velocity to be determined and $u_{\text {porous }}$ is given as $u_{\text {porous }}=-D_{a} \frac{d p}{d z}$ and

$$
R(z)=\left\{\begin{array}{lr}
1-2 \delta(z-d) ; & d<z \leq d+\frac{1}{2}, \\
1-\frac{\delta}{2}\left(1+\cos 2 \pi\left(z-d-\frac{1}{2}\right)\right) ; & d+\frac{1}{2}<z \leq d+1 \\
1 ; & \text { otherwise }
\end{array}\right.
$$

$D_{a}$ refers to the Darcy number which is employed to simulate the permeable wall.

\section{ANALYTICAL SOLUTION}

Solving eqs. (9) to (12) together with boundary conditions (13 a \& b), we obtain the solution for the velocity field, viz:

$$
\begin{aligned}
u(r, z)= & G_{r}(1-\varphi)^{5 / 2}\left(\frac{\varphi\left(\gamma_{\mathrm{s}} \rho_{\mathrm{s}}\right)}{\gamma}+(1-\varphi)\right)\left(\frac{1}{2} \Omega_{5}\left(r^{2}-R^{2}\right)+\frac{1}{4} \Omega_{6}\left(r^{4}-R^{4}\right)+\frac{1}{6} \Omega_{7}\left(r^{6}-R^{6}\right)\right) \\
& -\frac{\sqrt{D_{a}}\left(\left(\Omega_{10}\left(\frac{d p}{d z}\right) R / 2\right)+\alpha \sqrt{D_{a}}\left(\frac{d p}{d z}\right)\right)}{\alpha}+\left(\frac{d p}{d z}\right)(1-\varphi)^{5 / 2}\left(\frac{1}{4} \Omega_{3}\left(r^{2}-R^{2}\right)\right. \\
& \left.-\frac{1}{8} \Omega_{4}\left(r^{4}-R^{4}\right)\right),
\end{aligned}
$$

where the following definitions apply:

$$
\begin{aligned}
& \Omega_{1}=\frac{\beta}{4} \frac{k_{f}}{k_{n f}}, \quad \Omega_{2}=\frac{1}{4} \beta R^{2} \frac{k_{f}}{k_{n f}}, \quad \Omega_{3}=\Lambda \Omega_{2}+1, \\
& \Omega_{4}=\Lambda \Omega_{1}, \quad \Omega_{5}=\frac{\Omega_{3} \Omega_{2}}{2}, \quad \Omega_{6}=-\left(\frac{\Omega_{1} \Omega_{3}}{4}+\frac{\Omega_{2} \Omega_{4}}{2}\right), \\
& \Omega_{7}=\frac{\Omega_{4} \Omega_{1}}{4}, \quad \Omega_{8}=\frac{R^{2} \Omega_{3}}{24}-\frac{\Omega_{3}}{16}, \quad \Omega_{9}=\frac{G}{4}\left(\frac{R^{4} \Omega_{7}}{4}+\frac{R^{2} \Omega_{6}}{3}+\frac{\Omega_{5}}{2}\right), \\
& \Omega_{10}=(1-\varphi)^{5 / 2}\left(\Omega_{4} R^{2}-\Omega_{3}\right)-G_{r} R(1-\varphi)^{5 / 2}\left(\Omega_{5}+\Omega_{6} R^{2}+\Omega_{7} R^{4}\right)(\varphi(\gamma-1)+1) .
\end{aligned}
$$

The solution for the temperature field emerges as: 
$\theta(r, z)=\left(\frac{R^{2}-r^{2}}{4}\right)\left(\frac{k_{s}+2 k_{f}+2 \varphi\left(k_{f}-k_{s}\right)}{k_{s}+2 k_{f}-2 \varphi\left(k_{f}-k_{s}\right)}\right) \beta$.

The volumetric flux, $Q$, can be calculated as $Q=2 \int_{0}^{R} r u d r$.

The appropriate expression for axial pressure gradient is determined as:

$\frac{\partial p}{\partial z}=\frac{24\left(Q-\left(G_{r} R^{4}(1-\varphi)^{5 / 2}\left(6 \Omega_{5}+4 \Omega_{6} R^{2}+3 \Omega_{7} R^{4}\right)\left(\gamma_{\mathrm{f}} \rho_{\mathrm{f}}(\varphi-1)-\left(\gamma_{\mathrm{s}} \rho_{\mathrm{s}}\right) \varphi\right) / 24 \gamma_{\mathrm{f}} \rho_{\mathrm{f}}\right)\right)}{R^{2}\left(-24 \gamma-12 \sqrt{D_{a}} \Omega_{10} R / \alpha+R^{2}(1-\varphi)^{5 / 2}\left(2 \Omega_{4} R^{2}-3 \Omega_{3}\right)\right)}$.

Impedance resistance $\lambda$ is defined as $\lambda=\frac{\Delta p}{Q}$, since the flow rate $Q$ is constant for all sections of tube. Therefore impedance may be further modified as $\lambda=\frac{1}{Q} \int_{0}^{L}\left(-\frac{\partial p}{\partial z}\right) d z$ which yields:

$\lambda=\int_{0}^{d} F(z) d z+\int_{d}^{d+1 / 2} F(z) d z+\int_{d+1 / 2}^{d+1} F(z) d z+\int_{d+1}^{L} F(z) d z$

where $F(z)=-\frac{1}{Q} \frac{\partial p}{\partial z}$.

\section{RESULTS AND DISCUSSION}

Extensive numerical evaluation of the solutions derived in section 3 has been performed. On the basis of numerical evaluation, the computational graphs are illustrated in the Figs.2-6. The effects of pertinent parameters on velocity profile, temperature profile, wall shear stress, Impedance resistance, and streamlines are discussed in this section. 
Fig.2(a)

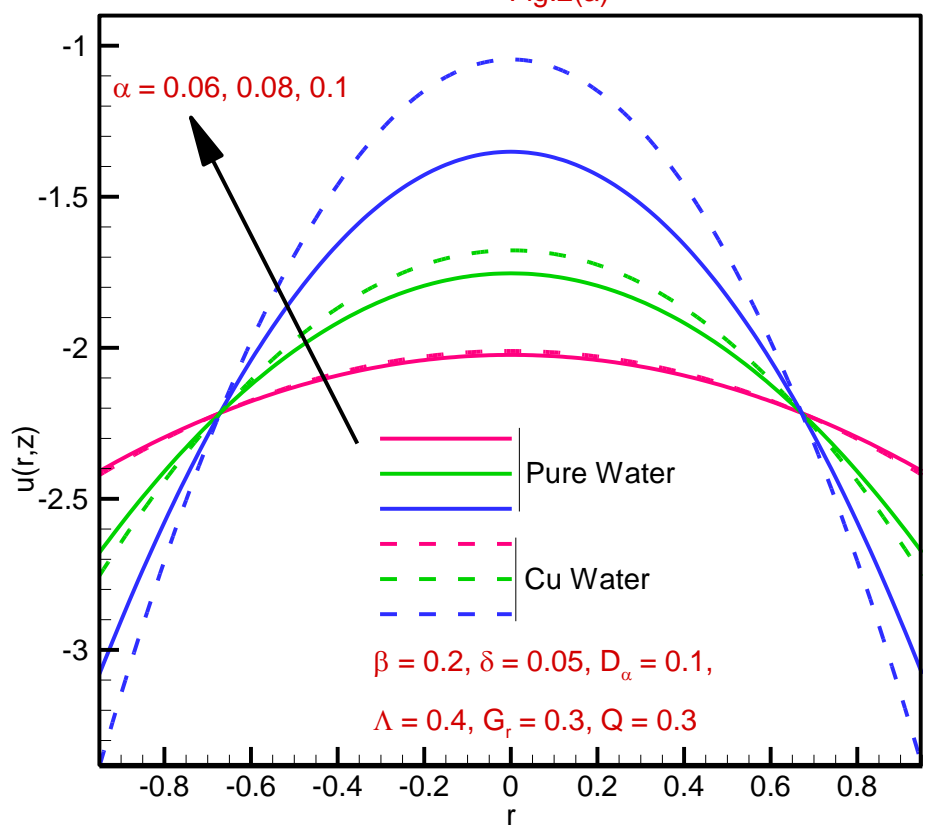

Fig. 2(b)

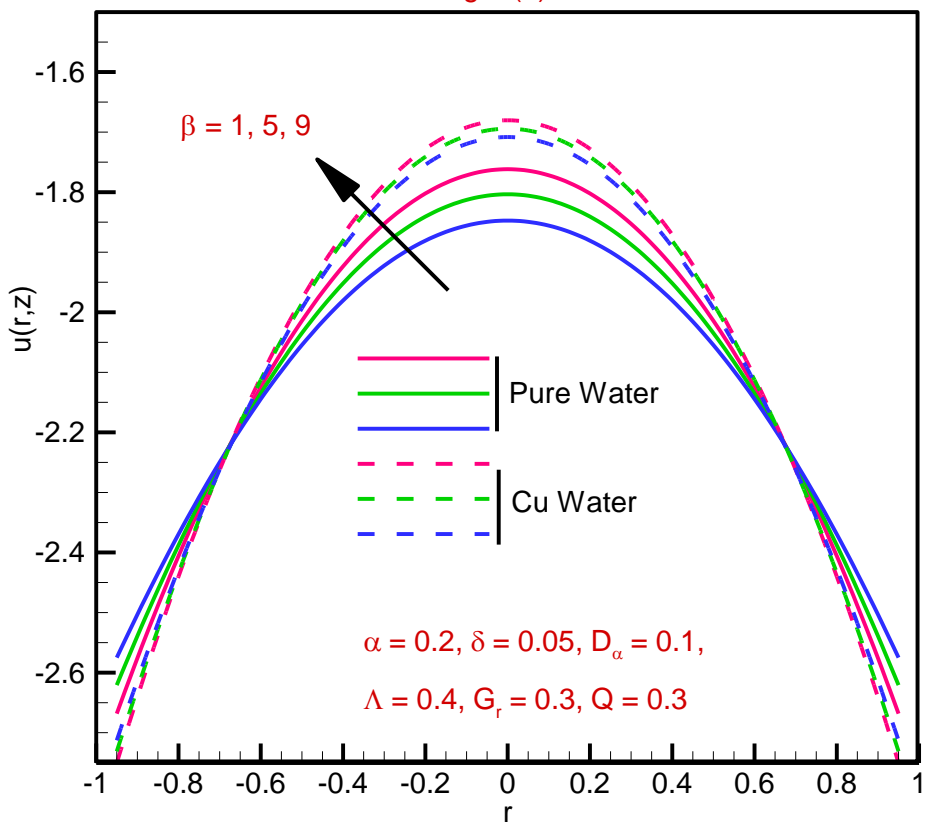



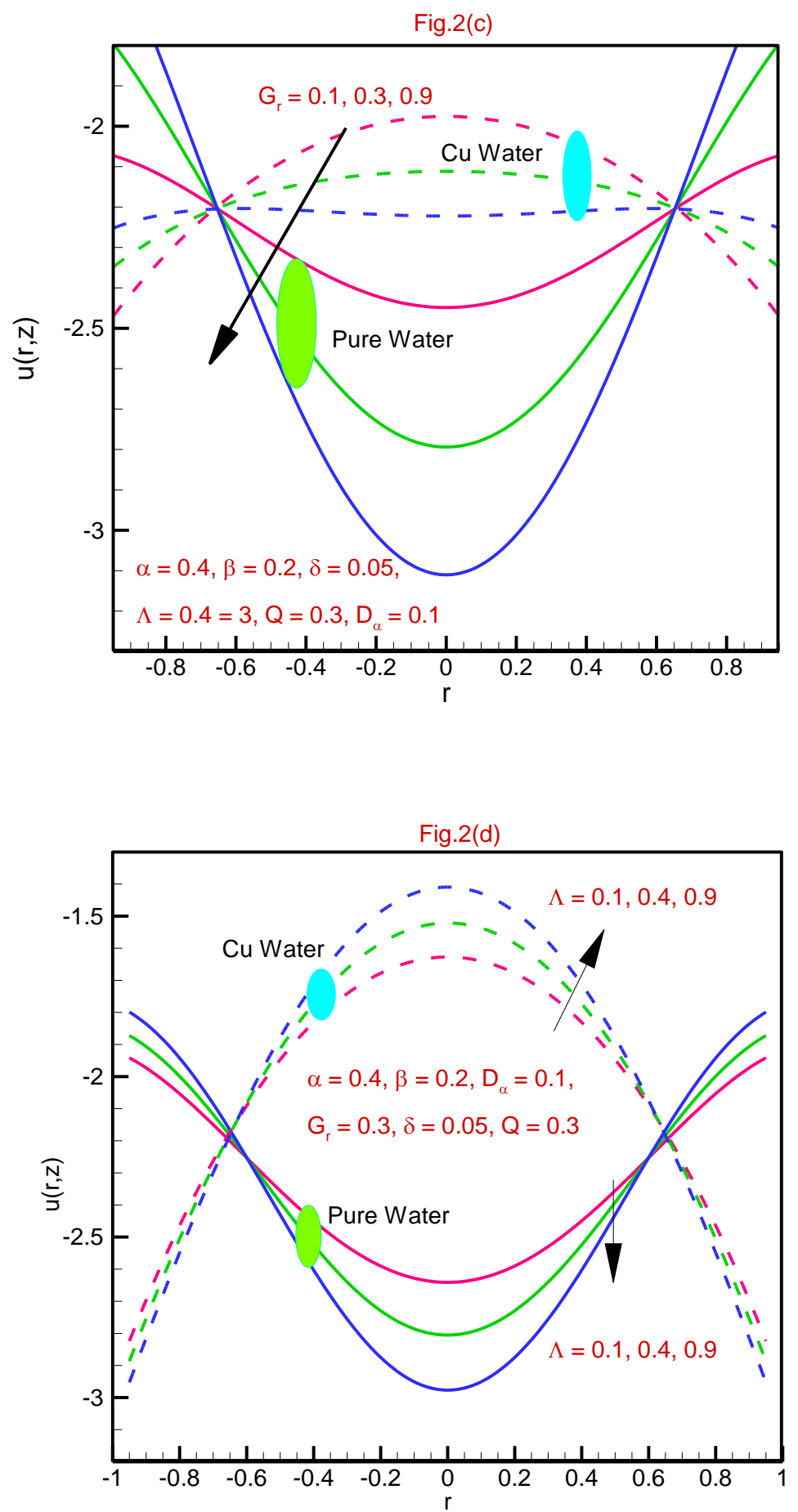

Fig.2. Velocity profile against the radial axis for different values of (a) Slip parameter, $\alpha$ (b) Heat absorption parameter, $\beta$ (c) Grashof Number, $G_{r}$ (d) Viscosity parameter, $\Lambda$. 

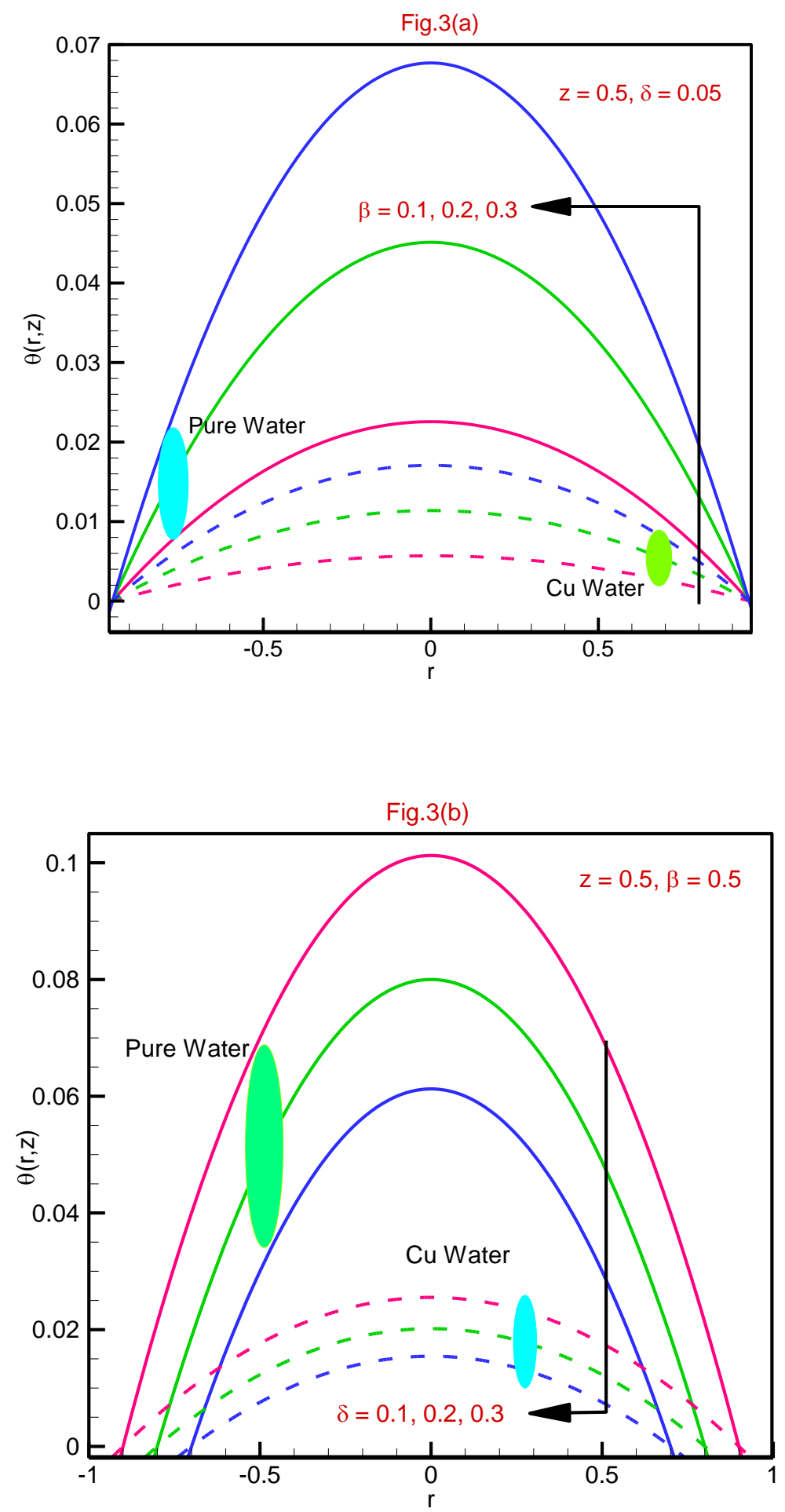

Fig.3. Temperature profile for different values of (a) Heat absorption parameter, $\beta$. Stenosis height, $\delta$. 
Fig.4(a)

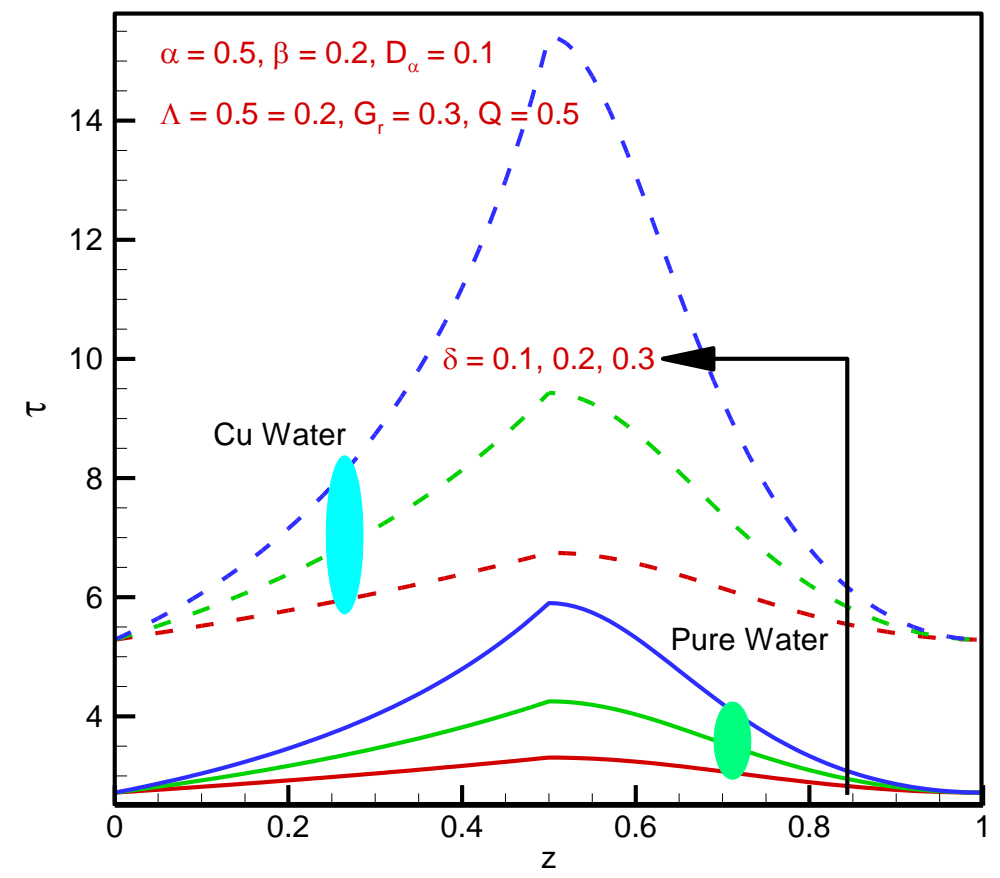

Fig.4(b)

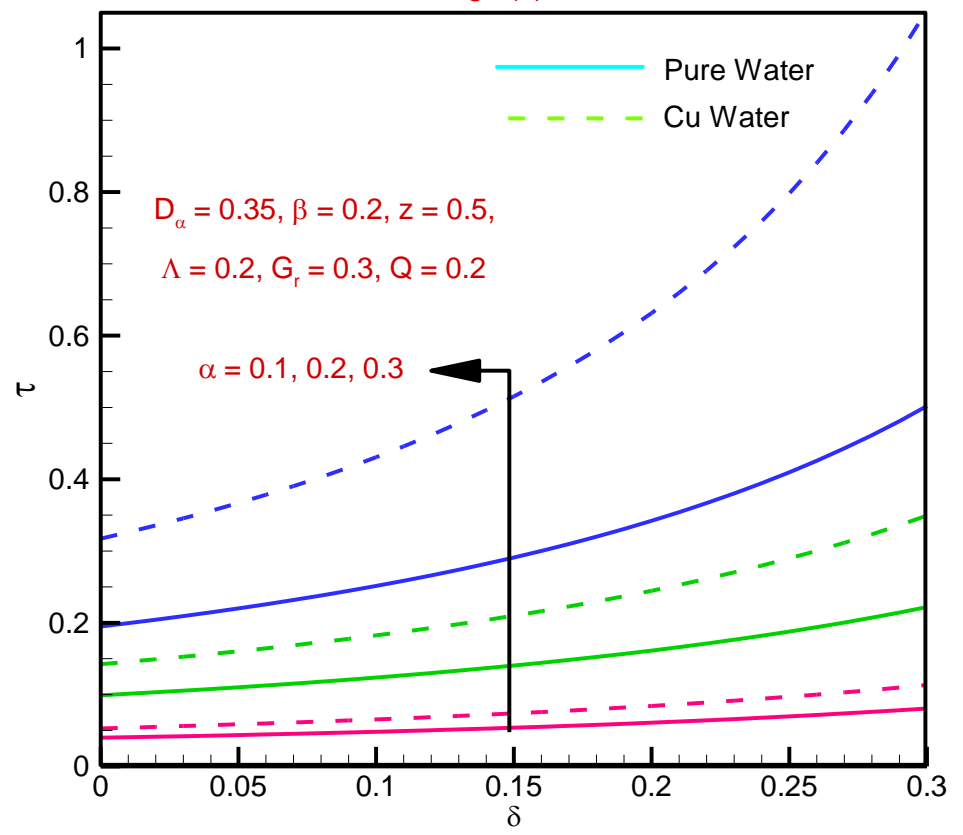




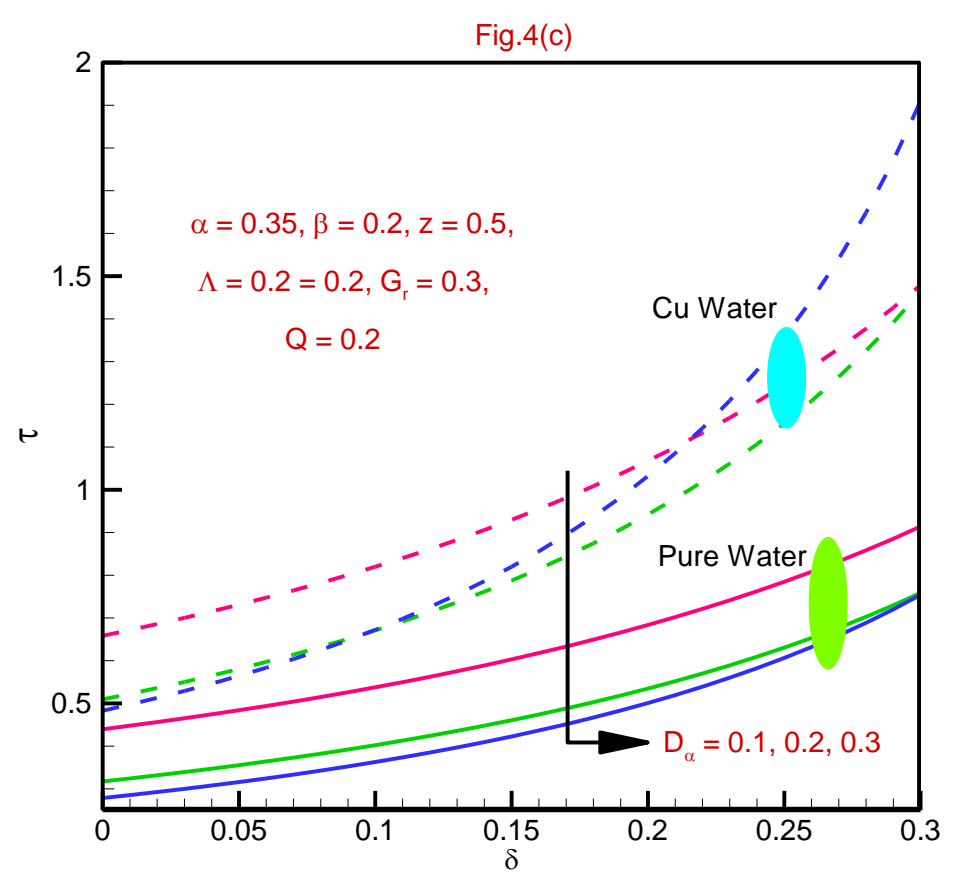

Fig.4. Wall shear stress for different values of (a) Stenosis height, $\delta$ (b) Slip parameter, $\alpha$ (c) Darcy number, $D_{a}$

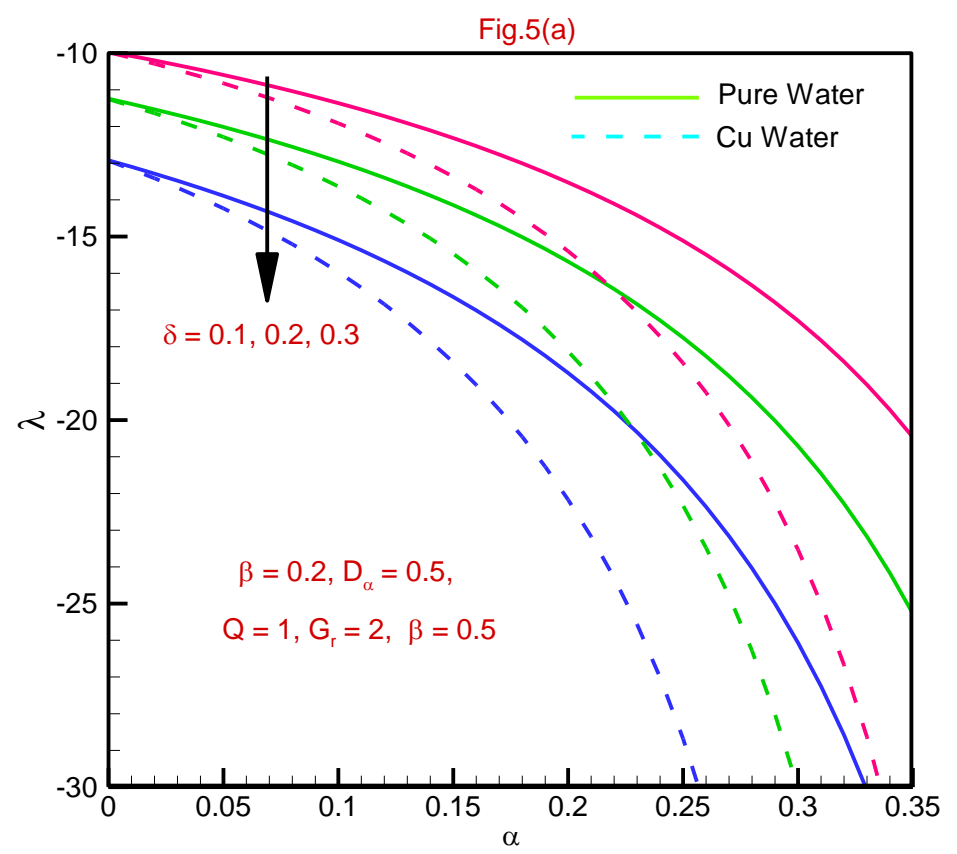




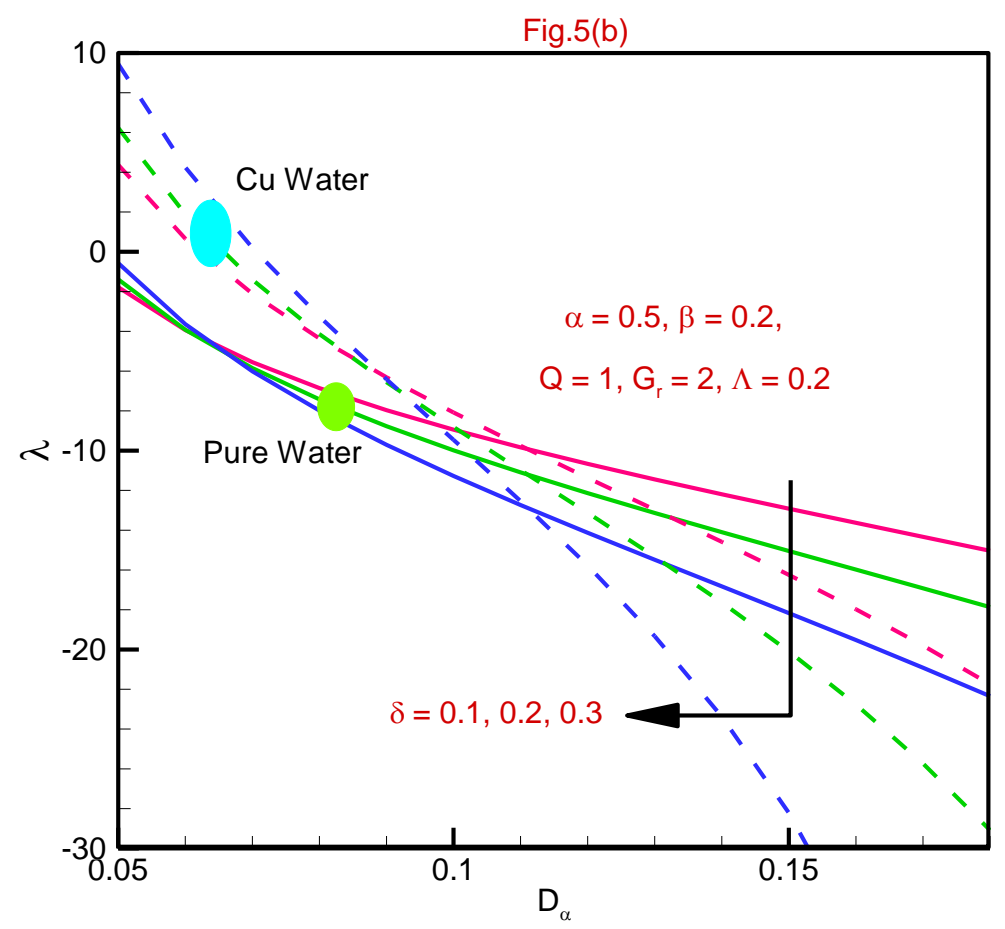

Fig.5. Impedance resistance for different values of (a) Stenosis height, $\delta$ (b) Darcy number, $D_{a}$

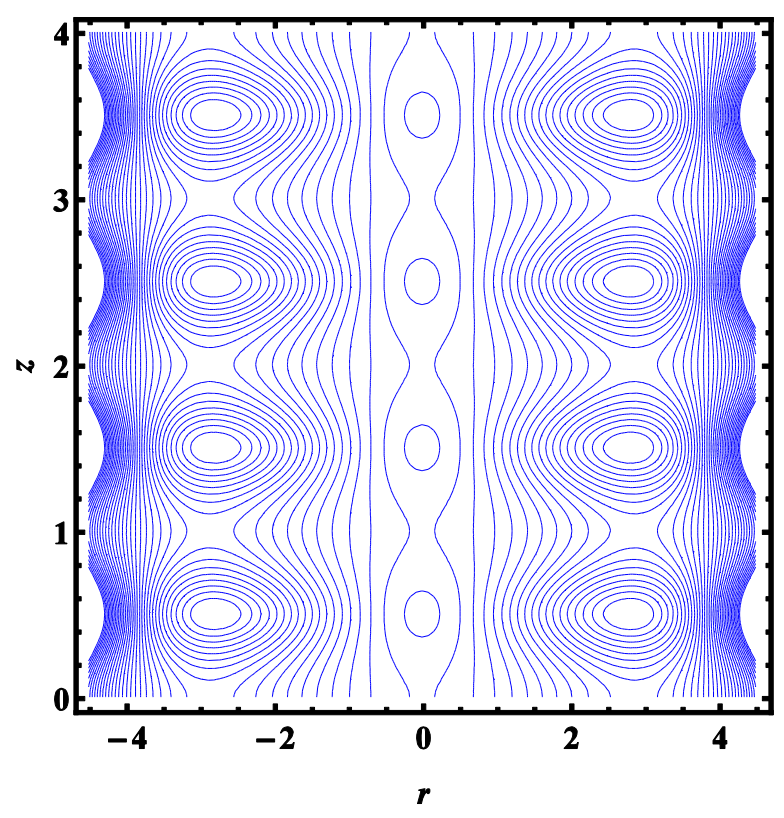

(a) Pure water $(\wedge=0.4)$

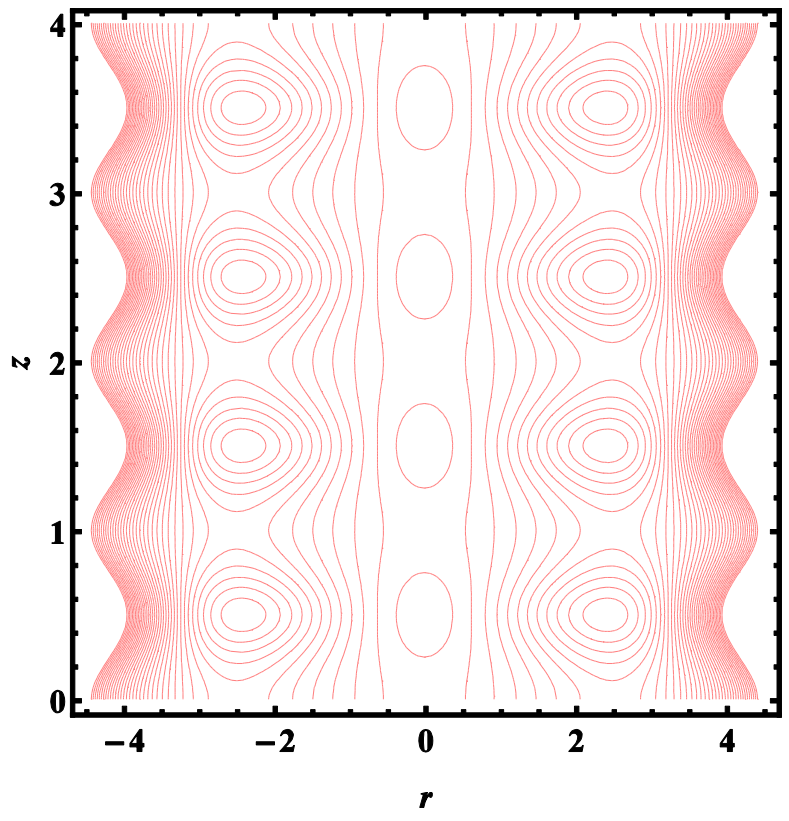

(b) Copper water $(\wedge=0.8)$ 


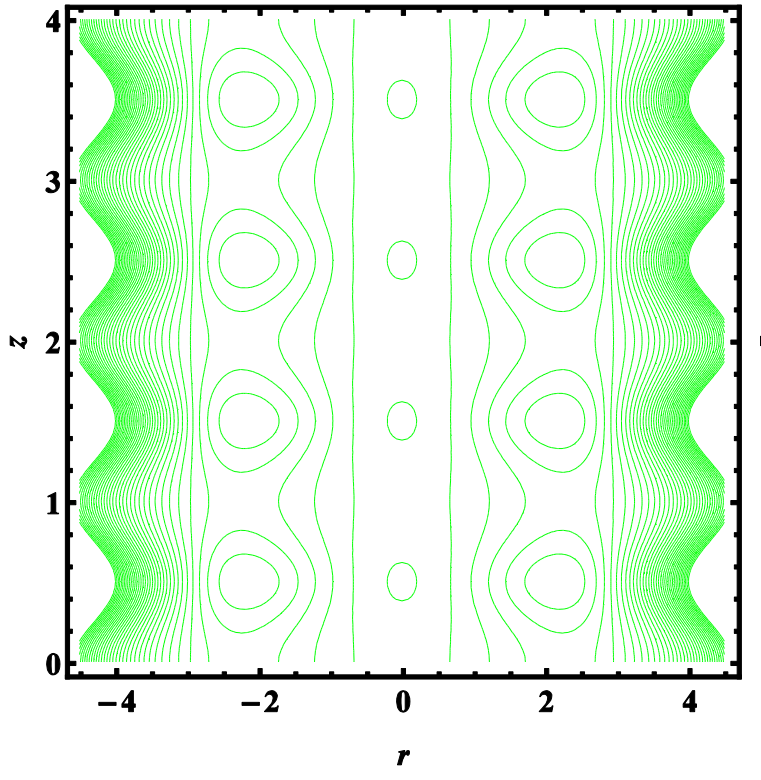

(c) Pure water $\left(G_{r}=1\right)$

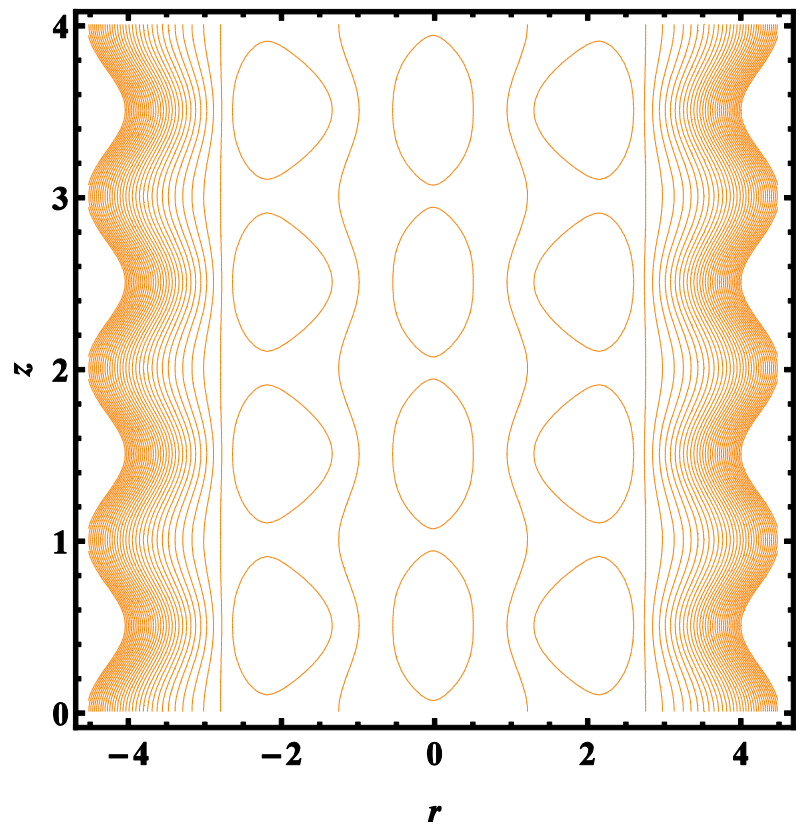

(e) Pure water $(\beta=1)$

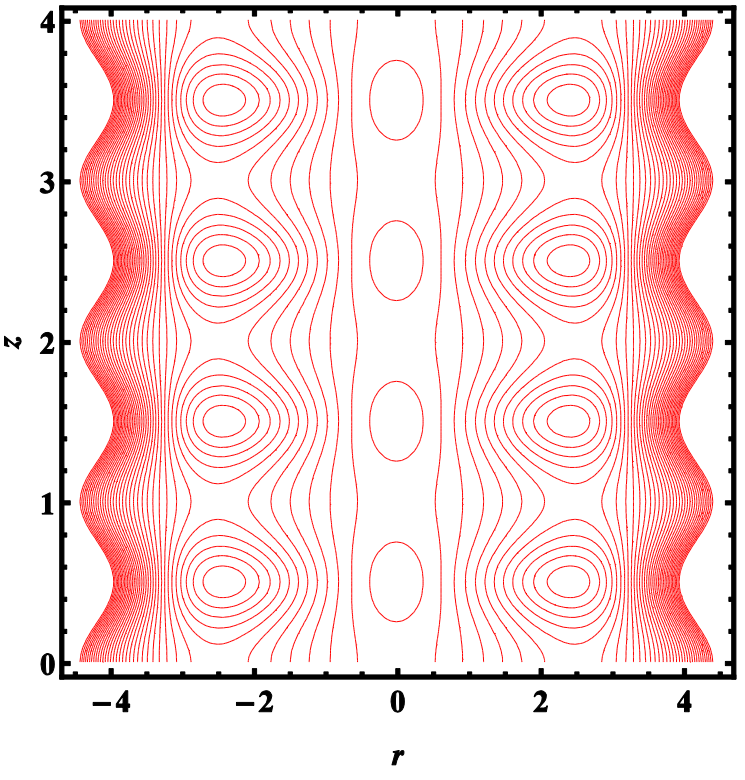

(d) Copper water $\left(G_{r}=2\right)$

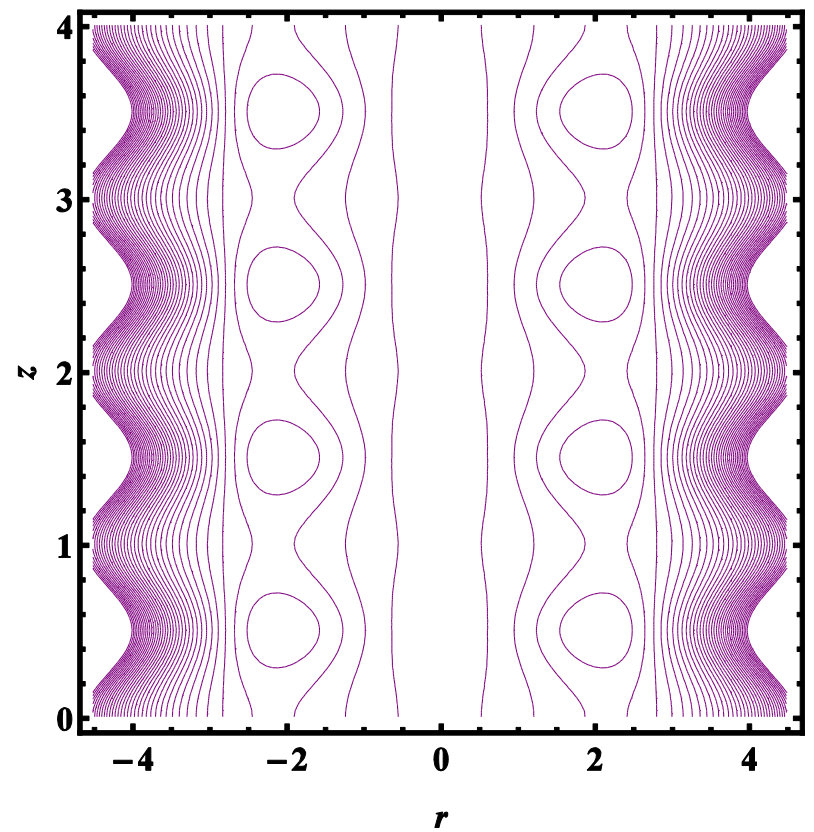

(f) Copper water $(\beta=3)$ 


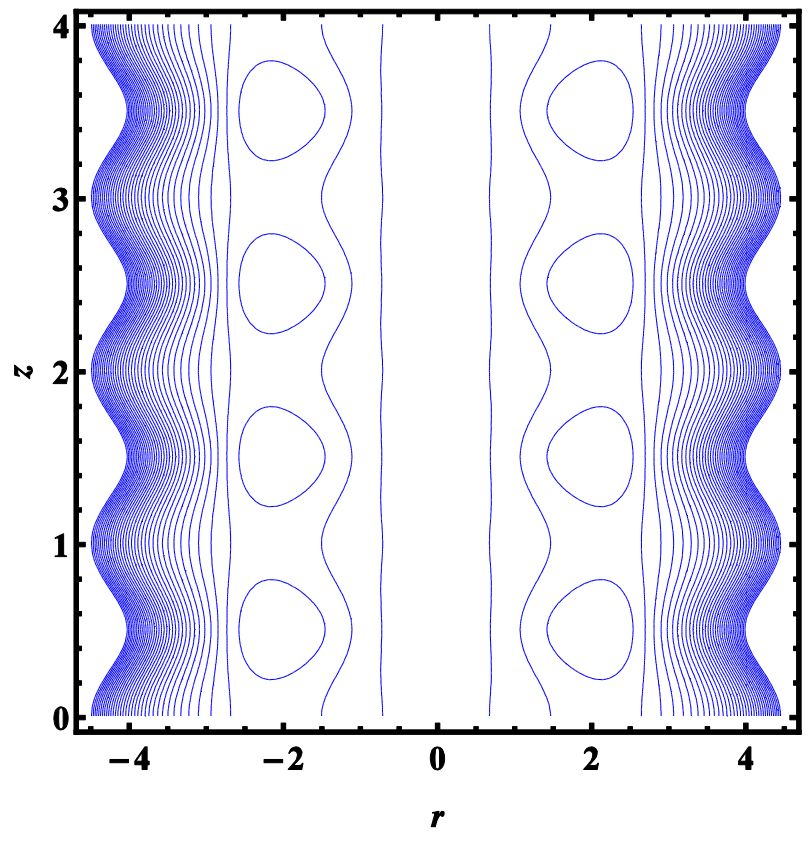

(g) Pure water $(D a=0.4)$

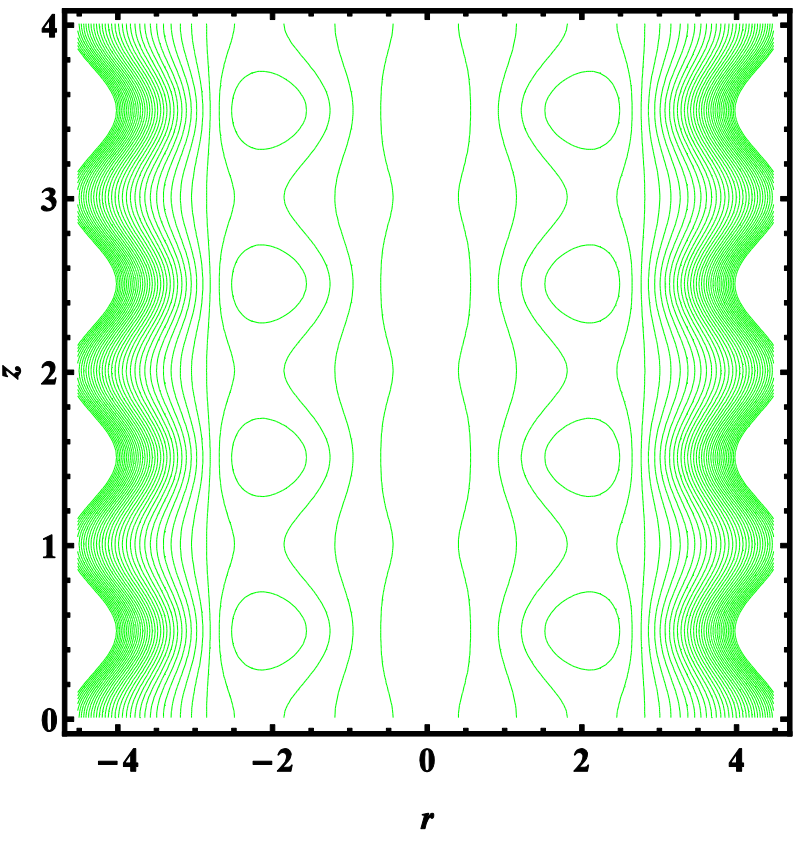

(h) Copper water $(D a=0.8)$

Fig.6. Streamlines for pure and copper water with different values of flow parameter i.e. (a)-(b) viscosity parameter, $\Lambda$ (c)-(d) Grashof number $G_{r}$ (e)-(f) Heat absorption parameter, $\beta$ (g)(h) Darcy number, $D_{a}$.

Fig.2 depicts the influence of selected parameters on the longitudinal (axial) velocity evolution, $u$ $(r, z)$ with radial coordinate $(r)$, for the two cases of pure water and copper water nanofluid. In all the profiles velocities grow from the lower radial coordinate (inner wall) to peak at the central core region, and thereafter diminish to zero at the outer permeable wall. With increasing slip parameter (fig. 2a) there is a marked acceleration in axial flow with increasing hydrodynamic wall slip effect. The slip parameter is only simulated via the boundary condition imposed at the inner surface of the outer permeable wall. The presence of slip inevitably induces a momentum boost at the zone of imposition which leads to elevation in velocities. Consistently the copperwater nanofluid achieves greater magnitudes than the pure water case, implying that the presence of copper nano-particles accelerates the blood flow. Absence of nanoparticles therefore leads to the converse effect i.e. hemodynamic deceleration. A similar observation has been computed by Nadeem and Ijaz [9], among others. The presence of nano-particles is therefore beneficial to the transport of blood. In fig. $2 \mathrm{~b}$ the presence of increasingly strong heat sink is observed also to generate substantial acceleration in the blood flow in the core region between the permeable 
walls, for both pure water and copper water nanofluid. However the contrary behavior is evident near the walls where lesser thermal energy introduced via the heat absorption is found to weakly decelerate axial velocity. The presence of a heat source may correspond clinically to, for example, a localized cooling of the artery via external mechanisms [37]. It works in the same way as a passive heat exchanger and due to the heat-conducting nature of real blood, can exert a significant role on temperature distributions also. The computations appear to be supported by actual clinical observations including the experiments of Petersen et al. [38]. The opposite effect i.e. heat source (thermal energy generation) is not considered due to limited applications in clinical hemodynamics. In the core region, copper water nanofluids attain higher velocities than pure water, with the reverse behavior computed in the proximity of the permeable walls. Fig. 2c illustrates the influence of the thermal buoyancy parameter i.e. Grashof number $\left(G_{r}\right)$ on axial velocity distributions. $G_{r}$ as defined in eqn. (8) effectively embodies the relative role of the thermal buoyancy (natural convection) force to the viscous hemodynamic force. For $G_{r}<1$ the viscous force dominates whereas for $G_{r}>1$ the thermal buoyancy force dominates. When $G_{r}$ equals unity both forces contribute equally. As $G_{r}$ increases the flow is observed to decelerate significantly, in particular in the core region. The values considered span from 0.1 to 0.9 and therefore for every profile there is a dominance of the viscous force over the thermal buoyancy force. This results in significant retardation of the axial flow. The converse effect may therefore be achieved with $G_{r}$ exceeding unity in which case thermal buoyancy will drive the axial flow with greater vigour and will lead to significant acceleration. Similar results have been reported for Newtonian viscous fluids by Abd elmabouda and Mekheimer [39]. The present solutions also show that copper nanofluids sustain greater accelerations in the core inter-wall region compared with pure water, even with low thermal buoyancy, again demonstrating the assistive nature of nano-particles to momentum transfer in hemodynamic transport. The impact of viscosity parameter, $\Lambda$, on velocity profiles is plotted in fig. $2 \mathrm{~d}$. The model used for viscosity variation, viz eqn. (12) i.e. $\frac{\mu_{n f}(\theta)}{\mu_{0}}=\frac{e^{-\Lambda \theta}}{(1-\phi)^{2.5}}, \quad e^{-\Lambda \theta}=1-\alpha \Lambda \theta+O\left(\Lambda^{2}\right), \quad$ is that of an exponentially decaying viscosity with temperature increase. It is also a function of nanoparticle volume fraction. Larger values of $\Lambda$, will therefore imply decreasing viscosity and this will lead to an acceleration in the blood flow for copper nanofluids, as testified to by the growth in profiles (dotted lines). However 
the converse effect is imparted to the pure water fluid case where with decreasing viscosity (increasing $\Lambda$ values) the blood flow is in fact decelerated. The presence of a volume fraction in the denominator in eqn. (8) results in greater effective values of viscosity at any value of $\Lambda$ for pure water compared with copper nanofluid. The presence of nano-particles therefore results in acceleration of blood flow at all values considered for viscosity parameter.

Figs.3a,b present the temperature evolution for various values of heat absorption and stenosis height, with radial coordinate. Immediately it is apparent that at the permeable walls only zero values of temperature arise, unlike the non-zero velocity values present in figs.2a-d. In the velocity plots, the wall slip results in the non-zero wall values, whereas since thermal slip is absent this characteristic does not arise in temperature distributions. Although thermal slip may be incorporated into the present model, we defer studying this to the future, since the present model contains numerous multi-physical effects as it is. With greater heat absorption, there is a strong elevation in temperatures. Symmetric profiles are generated across the inter-wall gap. Pure water is observed to however attain greater temperature magnitudes than copper water, since the nature of heat absorption is to remove thermal energy which the nano-particles achieve better than pure base fluid (water). The plots in fig.3a correspond to relatively low stenotic height $(\delta=0.05)$ and an intermediate axial location $(z=0.5)$, the latter being selected to be as representative of the general scenario as possible. In fig. $3 \mathrm{~b}$ an increase in stenotic height $(\delta)$ is found to significantly reduce the temperature values across the blood vessel radial dimension, and again smooth parabolic symmetric distributions are consistently computed. The copper water nanofluid again produces lower temperatures than the pure water with heat absorption (sink) effect present $(\beta=0.5)$.

Figs.4a-c illustrate the wall shear stress profiles for variation in various control parameters. Fig. 4a shows that increasing stenotic height is found to elevate the shear stress magnitudes and the peak shear stress surfaces at intermediate axial coordinate values $(\mathrm{z} \sim 0.5)$ Shear stress profiles are pinched and generally symmetrical about the peak value. With copper water nanofluid significantly greater wall shear stress is computed as compared with the pure water case. Fig. 4b demonstrates that shear stress is elevated significantly with wall slip parameter $(\alpha)$ and this is attributable to the acceleration in the axial flow. A steady growth in shear stress also accompanies increasing stenotic height values. Shear stress is minimized in the pre-stenotic 
region, implying that greatest flow deceleration arises here. With larger stenotic height, the shear stress will grow in the post-stenotic region where lesser impedance is experienced in the blood vessel. Generally copper-water nanofluid attains higher shear stress values than the pure water case. The influence of wall permeability on wall shear stress, as simulated via Darcy number $\left(D_{a}\right)$ is given in fig. 4c. With increasing Darcy number, the shear stress is observed to be decreased, implying that the flow is retarded. This is due to the greater influx of fluid into the vessel which destroys momentum and decelerates the flow. With increase in stenotic height, there is a strong growth in the wall shear stress at any Darcy number, for both copper water nanofluid and pure water.

Figs.5a-c depict the impedance resistance for different values of slip parameter, stenotic height and Darcy number. With increasing stenotic height the impedance magnitude is significantly elevated, as shown in fig. 5a. However it is also markedly increased with increasing slip parameter. Copper water nanofluid produces greater impedance magnitudes than the pure water fluid. Fig. 5b reveals that at low values of Darcy number, the impedance is initially greater for pure water as compared with copper water nanofluids compared with pure water. However at higher Darcy numbers, the opposite behavior is observed and copper water nanofluid achieves greater impedance magnitudes relative to pure water. Generally with increasing stenotic height, the impedance magnitudes are markedly elevated, as expected.

Figs.6a-h illustrate the streamlines in the $r-z$ plane for a variety of different parameters, and vortices are clearly visualized in these plots. In all the figures there are generally three lines of vortices, one along the longitudinal axis $(r=0)$ and two parallel streets of vortices on either side. The distributions are generally symmetrical about the line $r=0$ and are plotted along the entire length of the vessel $(0 \leq z \leq 4)$. With increasing viscosity parameter, $\Lambda$, there is a significant modification in the streamline plots in figs $6 a, b$. The central vortex region grows and the vortex size is enhanced pushing out into the dual parallel vortex streams on either side. Vorticity of the flow in the central region of the vessel is therefore intensified with greater viscosity parameter (lower effective viscosity of nanofluid). Figs $6 c$, d demonstrate that as the thermal buoyancy i.e. Grashof number, Gr, is increased from 1 to 2 (corresponding to a doubling in thermal buoyancy effect relative to viscous hemodynamic effect), the central vortex region is dramatically intensified and large vortices appear. Also there is a narrowing in streamlines in the parallel 
vortex streets on either side and vorticity is also intensified here. Buoyancy therefore exerts a very substantial effect on the hemodynamic process. The distortion to streamlines, in particular in the central zone is more severe than when viscosity is altered (figs 6a,b). Circulation is therefore more strongly affected by buoyancy than by viscosity effect. Figs 6e,f demonstrate that heat absorption $(\beta)$ has the opposite effect to increasing buoyancy. Instead of intensifying circulation in the central zone, vortices here are completely eliminated and the flow is intensified on either side. Finally with increasing Darcy number $\left(D_{a}\right)$ a similar effect is achieved in that the circulation zones along the $r=0$ line vanish and greater distortion in streamlines migrates outwards towards the parallel zones. The sensitivity of the hemodynamics to modifications in viscosity, buoyancy, heat absorption and wall permeability is therefore substantial and demonstrate the importance of including these multi-physical effects in realistic stenotic flow simulation.

\section{CONCLUSIONS}

A mathematical model has been developed to study variable-viscosity hemodynamic flow containing nano-particles (copper) and heat transfer in a vertical composite stenosed artery with permeable walls. Slip effects at the permeable walls of the blood vessel have been incorporated and also thermal buoyancy effects arising from density differences. The problem has been motivated by possible applications in nano-particle drug delivery in stenotic flows using a more realistic viscosity-variation model. Closed-form solutions for the transformed boundary value problem have been derived. A parametric study of the influence of copper nano-particles (via volume fraction), viscosity, heat absorption, stenotic height, slip parameter and Grashof number on velocity, temperature, wall shear stress, impedance resistance and also stream-line distributions, has been conducted. The present analysis has shown that:

- Greater buoyancy and reduced viscosity effect results in intensification of vorticity along the axis of the vessel, whereas greater wall permeability and heat absorption manifest in a decrease in circulation along the vessel longitudinal axis.

- An increase in wall shear stress is induced with greater stenotic height whereas temperature magnitudes are decreased.

- An elevation in heat absorption generates a strong boost in blood temperatures. 
- Increasing thermal buoyancy i.e. Grashof number, decelerates the core flow.

- Greater hydrodynamic wall slip strongly accelerates the flow in the core region.

- For copper water nanofluids the flow is accelerated in the core region compared with pure water.

\section{REFERENCES}

1. $\quad$ S. Berger and Jou L.-D., Annual Review of Fluid Mechanics. 32, 347 (2000)

2. J. Misra, Patra M., and Misra S., Journal of biomechanics. 26, 1129 (1993)

3. R. Ellahi, et al., Appl. Math. Inf. Sci. 8, 1567 (2014)

4. $\quad$ N.S. Akbar, et al., International Journal of Thermal Sciences. 85, 54 (2014)

5. $\quad$ N.S. Akbar, et al., The European Physical Journal Plus. 132, 1 (2017)

6. K.S. Mekheimer, Haroun M.H., and El Kot M., Canadian Journal of Physics. 89, 201 (2011)

7. R. Ellahi, Ur-Rahman S., and Nadeem S., Zeitschrift für Naturforschung A. 68, 489 (2013)

8. S. Chakravarty and Sannigrahi A.K., International journal of engineering science. 36, 1083 (1998)

9. $\quad$ S. Nadeem and Ijaz S., Physics Letters A. 379, 542 (2015)

10. L. Back, et al., Journal of Biomechanics. 19, 1 (1986)

11. U.S. Chakraborty, Biswas D., and Paul M., Korea-Australia Rheology Journal. 23, 25 (2011)

12. A.A. Owida, Do H., and Morsi Y.S., Computer methods and programs in biomedicine. 108, 689 (2012)

13. G. Schneiderman, Mockros L.F., and Goldstick T.K., Journal of biomechanics. 15, 849 (1982)

14. K.S. Mekheimer and El Kot M., Engineering Science and Technology, an International Journal. 18, 452 (2015)

15. N. Sher Akbar and Khan Z., International Journal of Biomathematics. 8, 1550026 (2015)

16. F. Mollica, Jain R.K., and Netti P.A., Microvascular research. 65, 56 (2003)

17. V.K. Joshi, et al., The European Physical Journal Plus. (2017) Accepted

18. R. Manimaran, World Academy of Science, Engineering and Technology. 5 (2011)

19. M.G. Rabby, Shupti S.P., and Molla M.M., Journal of Fluids. 2014 (2014)

20. N.S. Akbar, Huda A.B., and Tripathi D., The European Physical Journal Plus. 131, 332 (2016)

21. M.M. Molla and Paul M., Medical engineering \& physics. 34, 1079 (2012)

22. A. Zaman, et al., International Journal of Heat and Mass Transfer. 95, 1084 (2016)

23. P. Neofytou and Drikakis D., International journal for numerical methods in fluids. 43, 597 (2003)

24. D. Tripathi, Yadav A., and Anwar Bég O., The European Physical Journal Plus. 132, 1 (2017)

25. J. Tan, Thomas A., and Liu Y., Soft matter. 8, 1934 (2012)

26. D.E. Owens and Peppas N.A., International journal of pharmaceutics. 307, 93 (2006) 
27. M. Lundqvist, et al., Proceedings of the National Academy of Sciences. 105, 14265 (2008)

28. H. Otsuka, Nagasaki Y., and Kataoka K., Advanced drug delivery reviews. 55, 403 (2003)

29. K.N. Sugahara, et al., Cancer cell. 16, 510 (2009)

30. J. Misra and Schit G., Journal of Mechanics in Medicine and Biology. 7, 337 (2007)

31. M. Akbarzadeh, et al., Journal of Molecular Liquids. 220, 1 (2016)

32. M. Sheikholeslami, The European Physical Journal Plus. 132, 55 (2017)

33. J. Esfahani, et al., International Journal of Heat and Mass Transfer. 109, 1162 (2017)

34. S. Rahman, et al., Journal of Molecular Liquids. 218, 484 (2016)

35. M. Bhatti, Zeeshan A., and Ellahi R., Microvascular Research. 110, 32 (2017)

36. P. Joshi, Pathak A., and Joshi B., Applications and Applied Mathematics. 4, 343 (2009)

37. M.A. Ashburn, et al., The Journal of Pain. 4, 291 (2003)

38. K.K. Petersen, et al., International journal of physiology, pathophysiology and pharmacology. 3, 236 (2011)

39. K.S. Mekheimerc, Zeitschrift für Naturforschung A. 67, 185 (2012) 